FORMATION Formation emploi

Revue française de sciences sociales

100 | octobre-décembre 2007

De la formation professionnelle en Suisse

\title{
La formation comme politique d'activation des chômeurs en Suisse : divergences d'interprétation
}

Vocational training policies for activating job-hunters in Switzerland: divergent points of view

Ausbildung als Aktivierungspolitik für Arbeitslose in der Schweiz :

Auslegungsunterschiede

Olivier Giraud

\section{OpenEdition}

Journals

Édition électronique

URL : http://journals.openedition.org/formationemploi/1278

DOI : 10.4000/formationemploi.1278

ISSN : 2107-0946

Éditeur

La Documentation française

Édition imprimée

Date de publication : 1 octobre 2007

Pagination : $93-108$

ISSN : 0759-6340

Référence électronique

Olivier Giraud, « La formation comme politique d'activation des chômeurs en Suisse : divergences d'interprétation ». Formation emploi [En ligne], 100 l octobre-décembre 2007, mis en ligne le 01 octobre 2009, consulté le 30 octobre 2020. URL : http://journals.openedition.org/formationemploi/1278 ; DOI : https://doi.org/10.4000/formationemploi.1278 


\title{
DOSSIER
}

\section{La formation comme politique d'activation des chomeurs en Suisse : divergences d'interprétation'}

Par Olivier Giraud*

\begin{abstract}
Les politiques de l'emploi en Suisse constituent une illustration éclairante de la complexité des interactions multiniveaux qui caractérisent la plupart des régulations de politiques publiques contemporaines. Cette complexité est liée

à une diversité des perceptions, des débats, des équilibres politiques

qui fonde des mises en œuvre plurielles des politiques publiques.
\end{abstract}

Le système politique suisse, connu pour être l'un des plus décentralisés du monde occidental, permet d'analyser la façon dont des systèmes d'acteurs territorialisés réinterprètent des normes définies dans une arène centralisée. Cette question est essentielle dans les pays fédéraux, mais elle gagne de l'importance partout en Europe au fur et à mesure que les différents systèmes institutionnels se décentralisent. $\mathrm{La}$ France connaît, en la matière, un rattrapage important dans les domaines de la formation et de l'emploi

\footnotetext{
${ }^{1}$ Je remercie Jean-Claude Barbier pour sa lecture attentive d'une version antérieure de ce texte ainsi que les rapporteurs anonymes de «Formation Emploi » pour leurs remarques stimulantes. Les résultats de recherche présentés ici ont été financés dans le cadre du Programme National de Recherche $n^{\circ} 43$ du Fonds national de la recherche scientifique (Berne), intitulé « Formation et emploi ». $\mathrm{La}$ recherche fait l'objet d'une présentation détaillée dans l'encadré 1. Monica Battaglini (Institut d'études sociales, Genève), Virgile Perret (Institut d'études politiques et internationales - université de Lausanne) et Marc Helbling (Institut für Politikwissenschaft-Université de Zurich) ont contribué de façon décisive au projet tant par la contribution à la recherche empirique que par l'élaboration analytique et l'interprétation des résultats.
}

(Bel, Méhaut, Mériaux, 2003 ; Berthet, 2005). La constitution d'un niveau supranational de régulation en Europe renforce encore l'intérêt du cas suisse. La configuration politique et institutionnelle de ce pays constitue en l'occurrence une évocation intéressante du processus de construction d'une forme d'État fédéral européen. La Suisse est en effet un système fédéral décentralisé, constitué sur la base d'une multiplicité de clivage - linguistiques, religieux,

* Olivier Giraud est politologue, CNRS/Centre Marc Bloch, Berlin. Il a notamment publié en 2007 : "Processus d'institutionnalisation de la démocratie industrielle et crises sociales en France et en Allemagne à la fin des années 60 ", Travail et Emploi, n 11 1, pp. 39-52 (en collaboration avec Michèle Tallard et Catherine Vincent). Les cantons suisses face au chômage - Fédéralisme et politiques de l'emploi, L'Harmattan, Collection "Logiques Sociales », Paris (en collaboration avec Virgile Perret, Marc Helbling et Monica Battaglini). 
politiques, ville/campagne, etc. - et caractérisé par de fortes disparités politiques, économiques et sociales.

Comme c'est d'ailleurs le cas dans l'Union européenne (Barbier 2005), les nouvelles politiques de l'emploi en Suisse se réfèrent largement à la notion d'activation. Le caractère polysémique de cette notion est souvent perçu comme une imprécision, ouvrant la voie à des ambiguïtés, voire à des contradictions. Les politiques suisses de l'emploi, comme cela a pu être observé aussi dans le contexte européen (Barbier, 2004), donnent ainsi lieu à des divergences d'interprétation qui se révèlent notamment à travers une analyse de la mise en œuvre, dans les différents contextes régionaux, des dispositifs définis au niveau central. Ces divergences d'interprétation débouchent cependant souvent sur la définition d'un sens partagé, d'un bon sens à usage local ou régional. Des interprétations dominantes - « les femmes ne peuvent pas exercer des métiers d'homme», «les niveaux scolaires sont en baisse constante », etc. - n'ont même plus à être fondées ou justifiées. Ces raccourcis deviennent alors des fondements importants de l'action, notamment s'ils ne rencontrent qu'une faible opposition au sein des réseaux administratifs et sociaux pertinents d'un secteur d'intervention. L'analyse de ces sens communs locaux dans le cas de la formation comme politique d'activation des chômeurs fournira une illustration de ce mécanisme.

La question de la variabilité potentielle des mises en œuvre des politiques publiques, par exemple sous l'influence des agents de terrain (street-level bureaucrats), est une question classique des études sur le fédéralisme (Pressman, Wildavsky, 1973). Elle se pose aujourd'hui de façon beaucoup plus large au sein des États fédéraux - Suisse, Allemagne, - et dans les entités institutionnelles de type fédéral - Union européenne... - mais aussi dans les États unitaires. Inspirées des théories de la régulation en sociologie ${ }^{2}$, les approches en termes de régulation politique (Jobert, 1998), permettent de bien comprendre comment les décisions politiques prises dans une arène politique centralisée - fédérale ou nationale - se rejouent à l'échelle régionale ou locale en fonction des

2 Pour une présentation récente de ces théories, $c f$. de Terssac, 2003. ressources, des traditions, des mobilisations ou des réseaux politiques (Knoepfel, Larrue, Varone, 2006).

Après une introduction aux spécificités du marché du travail et des réformes les plus récentes des politiques pour l'emploi de la Suisse, notre contribution examine de façon approfondie les variations constatées dans les modes de mise en œuvre des politiques pour l'emploi dans les différents cantons suisses, au début des années 2000. Nous conclurons la réflexion par l'examen des divergences d'interprétation qui se nouent, à propos de la formation, dans six cantons suisses. La restitution de ces divergences repose sur la confrontation de discours tenus par différents acteurs des politiques de l'emploi. Elle permet d'expliquer en partie les fortes divergences empiriquement constatées dans les usages de la formation professionnelle comme politique d'activation des chômeurs.

\section{MUTATIONS RÉCENTES DES POLITIQUES DE L'EMPLOI EN SUISSE}

Le marché du travail suisse est longtemps resté une exception dans le contexte ouest-européen. Apparemment épargné par la crise des années 70 , alors même que l'économie nationale avait détruit plusieurs centaines de milliers d'emplois, ce marché du travail se caractérisait par l'adaptation de la demande de travail à l'offre. Le chômage n'augmentait pas en Suisse pour deux raisons principales : d'une part, les femmes, nouvelles venues et peu légitimes dans l'emploi, et les étrangers, à l'époque rarement titulaires de permis d'établissement ${ }^{3}$, occupaient des positions si fragiles sur le marché du travail qu'ils s'en retiraient en cas de perte d'emploi. D'autre part, l'absence de système uniforme et obligatoire d'indemnisation du chômage rendait l'inscription au chômage largement inutile. Progressivement, dans les années 80, la Suisse a procédé à des réformes qui ont normalisé la situation du système d'emploi du pays (Schmidt, 1995).

\footnotetext{
${ }^{3}$ Permis de travail qui permettent aux personnes de s'établir dans le pays à plus ou moins long terme.
} 


\section{Des rénovations tardives}

La situation des étrangers a tout d'abord été stabilisée sur le marché suisse du travail. Confronté à une série de critiques évoquant les pratiques «d'exportation du chômage » conduites par la Suisse, le pays a réformé ses politiques d'immigration. Aujourd'hui, la plupart des personnes qui travaillent en Suisse depuis plus de cinq ans bénéficient d'un permis d'établissement qui leur confère des droits, sur le marché du travail, équivalents à ceux des Suisses (Giraud, Braun, 1999). La place des femmes sur le marché du travail a par ailleurs fortement progressé, le taux d'activité féminin passant de 33 à $50 \%$ entre 1960 et 2003 (OFS, 2005). Enfin, une réforme cruciale a rendu l'assurance-chômage obligatoire pour tous les salariés, à partir de 1984.

Ces différentes réformes et transformations sont intervenues dans une période faste sur le marché du travail. Tout au long des années 80 , l'activité a redémarré en Suisse, le taux d'emploi s'est de nouveau hissé à des niveaux élevés en comparaison avec les pays de l'OCDE (Organisation de coopération et de développement économiques) et de nombreux étrangers se sont établis sur le marché du travail helvétique.

Les années 90 sont en revanche celles de la rupture. La crise économique qui a marqué le début de la décennie a entraîné, en Suisse, un changement de cycle brutal. Pour la première fois depuis les années 30 , le taux de chômage dépasse le seuil de $1 \%$ et même de $5 \%$ en 1995 ( $c f$. graphique 1). En dépit d'un fonctionnement du marché de l'emploi resté globalement dynamique (Bonoli, Mach, 2001), cette détérioration rapide révèle de nouvelles fragilités concernant à la fois le pourcentage de chômeurs de longue durée (plus d'un an de chômage), avoisinant les $30 \%$ au plus fort de la crise, mais aussi des disparités territoriales importantes. Dans les cantons de Suisse latine, qui sont également le plus souvent des cantons frontaliers, le taux de chômage est en effet largement au-dessus de cette moyenne. Ce taux a approché $8 \%$ dans les cantons de Genève et du Tessin en 1997, et $7 \%$ dans les cantons de Vaud, Valais, Jura et Neuchâtel.

Cette entrée en crise du marché du travail suisse, dans les années 90 , a incité le législateur fédéral à réformer les politiques de l'emploi. La loi de 1984 s'était contentée d'introduire une assurance-chômage obligatoire. Les politiques suisses pour l'emploi restaient des politiques passives, mises en œuvre au niveau communal. La plupart des communes ne faisaient que contrôler les chômeurs et instruire les dossiers de chômage en liaison avec les caisses d'assurance.

\section{Graphique 1}

Évolution du chômage en Suisse de 1990 à 2004

(Source : Service des Statistiques du marché du travail, Secrétariat d'État à l'Économie, Berne)

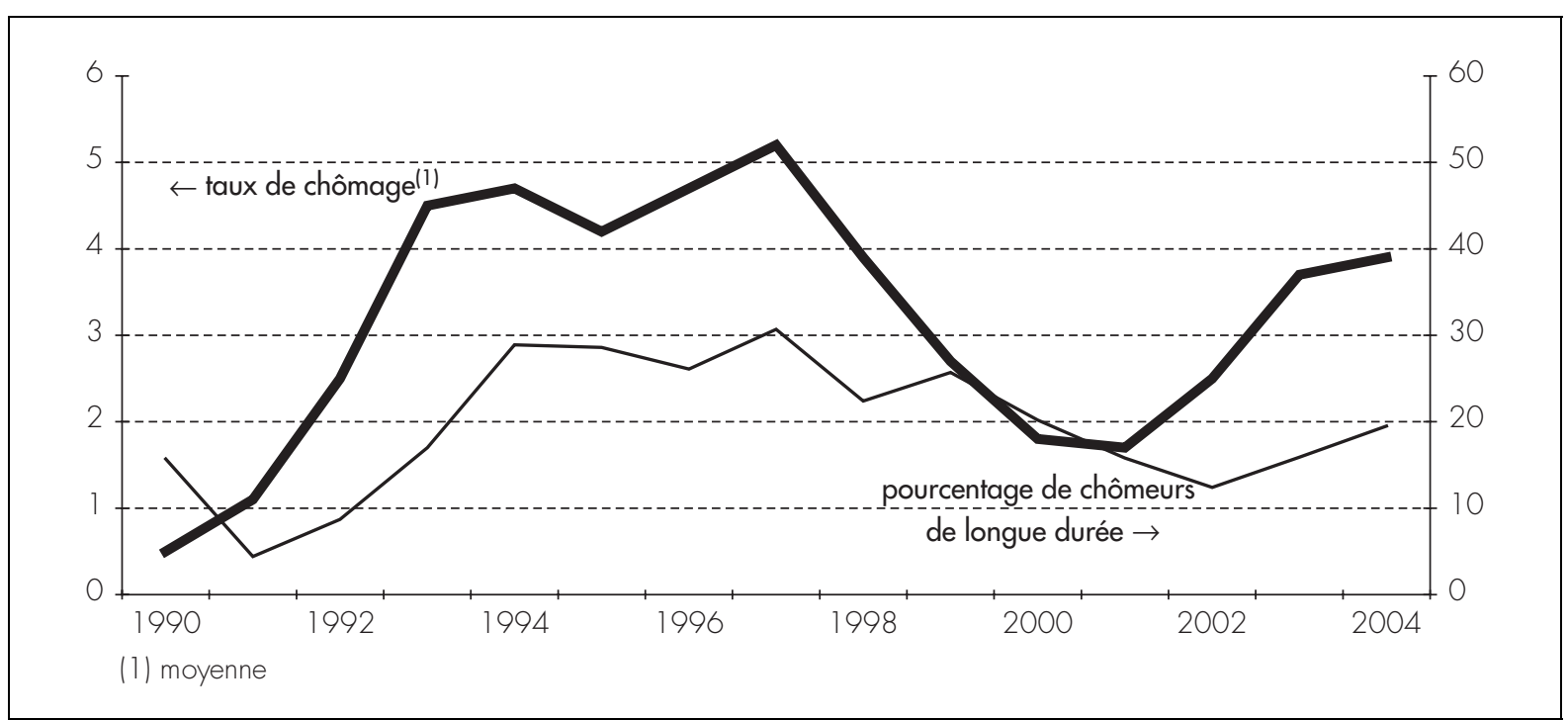


Au milieu des années 90, la seconde révision de la loi fédérale sur le chômage a apporté un grand nombre d'innovations sur le plan des politiques actives de l'emploi (Giraud, Braun, 1999). Au vu de la progression du chômage, le gouvernement fédéral suisse a souhaité mettre en conformité ses politiques pour l'emploi avec les recommandations de l'OCDE (1996). À cet effet, il a initié deux réformes radicales. Le gouvernement fédéral a d'abord décidé de mettre en œuvre des politiques actives de lutte contre le chômage, en accordant un rôle important à la formation. Il a également transformé totalement la mise en œuvre de ces politiques en organisant le transfert de la compétence en matière de politiques de l'emploi des communes aux cantons. La faiblesse financière et administrative d'un grand nombre de communes ne permettait en effet pas à ces dernières de remplir de façon efficace leurs attributions en la matière. Il est nécessaire de s'arrêter sur ces deux changements de fond.

\section{L'activation au cœur de la lutte contre le chômage}

Les nouvelles politiques suisses de l'emploi relèvent d'une démarche d'activation. L'activation des politiques pour l'emploi est une notion-fétiche du secteur qui correspond au moins à trois réalités différentes (Sylla, Barbier, 20064).

En premier lieu, l'activation est un objectif qualitatif et stratégique des politiques publiques de l'emploi ; elle est alors exprimée sous la forme d'un ratio financier qui correspond au montant des dépenses actives (formations, stages, emplois subventionnés) sur le montant des dépenses passives (indemnisation de la perte du revenu).

En deuxième lieu, l'activation est un objectif de politique publique destiné aux chômeurs. Il s'agit d'une obligation imposée aux personnes sans emploi. Afin de conserver leur indemnisation, elles doivent suivre une mesure «active» (stage, formation, emploi subventionné), destinée à renforcer leurs chances sur le marché du travail. De ce point de vue, l'activation

\footnotetext{
${ }^{4}$ Dans le cadre d'une analyse plus détaillée, Ndongo Samba Sylla et Jean-Claude Barbier pointent un nombre plus important d'acceptions de l'activation dans le champ des politiques de l'emploi.
}

est censée renforcer l'employabilité du chômeur en adaptant son profil professionnel aux demandes du marché du travail. Dans le cas suisse, l'activation doit intervenir, en règle générale, six mois après l'entrée au chômage.

En troisième lieu, l'activation est un mot-clé associé à des débats de nature plus polémique - ou tout au moins plus politique - dans le domaine des politiques pour l'emploi. Ce terme renvoie en effet à un impératif moral censé s'appliquer aux chômeurs. Ces derniers doivent être «actifs» sur le marché du travail, « activement» rechercher un emploi, « activer» ou " réactiver» leurs connaissances, compétences et réseaux, etc. Cet impératif de l'activation a été abondamment encouragé par l'OCDE, notamment à travers ses campagnes visant à renforcer les incitations au travail («Making Work Pay »). Il s'agit alors non seulement de maintenir ou développer un écart suffisant entre les revenus issus du travail et les revenus tirés des assurances chômage ou d'autres prestations sociales, mais aussi de décourager la passivité des personnes qui ne sont pas en emploi en exigeant des compensations (workfare).

\section{Un contrôle renforcé des chômeurs}

Derrière ces différentes formulations des impératifs d'activité se profilent, dans la plupart des pays, des procédures de contrôle des chômeurs. Souvent revendiquée comme un héritage de la social-démocratie suédoise (Svensson, 2001), l'alliance entre activation et contrôle renvoie de fait à un compromis politique. Les décideurs publics acceptent de dépenser plus au profit de l'insertion du chômeur - par exemple en recourant à des mesures de formation - mais ils exigent en contrepartie un contrôle renforcé de l'utilisation des dépenses supplémentaires occasionnées. L'accord consistant à renforcer les mesures d'insertion en échange d'un accroissement des possibilités de contrôle des chômeurs s'est noué explicitement dans ces termes au sein des chambres fédérales suisses (Giriens et al., 1999).

Cependant, même si les programmes d'activation sont souvent conçus comme des ensembles de mesures qui supposent d'agir à la fois sur le volant insertion, sur le volant indemnisation et aussi sur le volant placement des chômeurs, de nombreux auteurs 
s'accordent à dire qu'il est possible de distinguer des régimes d'activation en fonction des inflexions qui sont données aux différentes mesures et à leurs inspirations. Ainsi, alors que Heikkilä (1999) oppose une version libérale-progressiste et une version plus directement « punitive » des régimes d'activation, Barbier (2004) isole pour sa part un régime libéral et un régime social-démocrate d'activation. Cette proposition conduit à intégrer les mesures spécifiques de lutte contre le chômage - et même celles qui sont directement susceptibles d'être rattachées à une stratégie d'activation - dans un ensemble de caractéristiques analytiques qui renvoient à la cohérence sociétale des contextes nationaux: les valeurs et normes dominantes des systèmes de protection sociale, les systèmes de relations professionnelles, les régimes d'emploi et d'activité par exemple (Ibid).

De ce point de vue, la Suisse est un cas difficile à caractériser. Manfred Schmidt (1995) évoque, dans le cas de la Suisse, un régime socio-économique et d'État providence « social-libéral ». Giuliano Bonoli et André Mach (2001) parlent pour leur part d'un régime d'emploi et d'activité qui se décline de façons différenciées en fonction des grands secteurs d'activités. Selon les catégories en termes de variétés de capitalisme développées par Hall et Soskice (2002), dans le secteur industriel, la Suisse serait ainsi plutôt proche de l'idéal-type de l'économie coordonnée; les régulations conventionnelles puissantes, les relations de travail stables, le poids de l'apprentissage industriel selon le modèle dual ou encore la nature des relations entre les banques et les entreprises sont autant d'indicateurs du rattachement à ce modèle. En revanche, dans les secteurs des services, le régime suisse d'emploi est beaucoup plus proche de l'idéaltype de l'économie de marché non coordonnée : les syndicats et les régulations conventionnelles sont faibles et le marché du travail est flexible.

\section{Un compromis politique dans la loi de 1995}

Les mesures d'activation contenues dans la loi de 1995 résultent d'un compromis politique entre droite et gauche parlementaires, qui a largement influencé l'équilibre entre mesures de réinsertion et mesures de contrôle des chômeurs.
Dans le contexte suisse, l'activation renvoie à une première disposition : après six mois de chômage, les personnes à la recherche d'un emploi doivent pouvoir accéder à une mesure active. Cette disposition correspond précisément au bréviaire contemporain des recommandations en matière de politiques de l'emploi, qu'elles émanent de l'OCDE ou de l’Union européenne.

Ensuite, la palette des mesures actives censées favoriser la réintégration des chômeurs sur le marché du travail est assez large (SECO, 2006). Elle regroupe en effet des mesures de soutien à la prise d'un emploi en dehors de la région de domicile, des mesures d'encouragement à une activité indépendante, des emplois temporaires subventionnés et enfin, différentes mesures de formation. Ces dernières sont d'ailleurs les plus utilisées par l'administration de l'emploi. Il s'agit à la fois de mesures de reconversion, de perfectionnement et d'intégration professionnelle. Enfin, certaines mesures sont plus particulièrement destinées aux personnes très éloignées de l'emploi ; ce sont des mesures de re-mobilisation ou d'aide au placement (rédaction de CV, préparation aux entretiens ou aux tests, etc.).

La loi fédérale sur le chômage a également introduit un arsenal de mesures permettant de contrôler et sanctionner les chômeurs pour différents motifs. Il est ainsi possible de prononcer des sanctions à l'encontre des demandeurs d'emploi jugés insuffisamment actifs sur le marché du travail, mais aussi à l'encontre des chômeurs qui ne respectent pas les règlements et procédures du service public de l'emploi. Les sanctions prennent la forme de pénalités graduelles de non-paiement de journées d'indemnisation du chômage. Les sanctions les plus lourdes doivent faire l'objet de discussions ou d'agrément par les directions des ORP (Offices régionaux de placement). Dans certains cantons, comme dans le canton de Berne, un médiateur ou une commission permettent des recours.

La conception de l'activation contenue dans les politiques suisses pour l'emploi est ainsi complexe dans la mesure où elle contient simultanément les trois dimensions attachées à ce concept. Premièrement, le mécanisme de l'octroi automatique d'une mesure d'activation à toute personne au chômage depuis plus 
de six mois entraîne une augmentation automatique de la part des dépenses actives dans le total des dépenses pour l'emploi. Deuxièmement, l'activation obligatoire des chômeurs influe directement sur les modalités d'insertion des chômeurs. Enfin, dans le contexte suisse, la rigueur des mesures de contrôle indique que l'activation est aussi comprise comme un impératif, fondé moralement, qui s'adresse aux individus et qui conditionne leur comportement sur le marché du travail.

La pluralité des références implicites et explicites à la notion d'activation dans la législation suisse des politiques pour l'emploi laisse le champ libre à des interprétations diverses. Certains insistent sur le caractère contradictoire d'une loi qui juxtapose des instruments de réinsertion active et des instruments de contrôle des chômeurs. Cette ambivalence se manifeste potentiellement dans la relation entre chômeurs et conseillers-placeurs du service de l'emploi. Les uns comme les autres sont en effet susceptibles de se trouver pris en contradiction entre une temporalité courte, celle des impératifs du contrôle et de ses nécessaires validations régulières, et une temporalité longue qui s'établit en fonction des nécessités de formation ou de reconversion des demandeurs d'emploi (Magnin, 2004).

De son côté, le Secrétariat d'État à l'Économie (SECO), administration fédérale de référence en matière de politiques de l'emploi, justifie la juxtaposition de ces instruments au motif que la durée de chômage est un facteur important d'aggravation de la situation des chômeurs. Du point de vue de l'administration fédérale, il faut ainsi tout mettre en œuvre pour accélérer le placement des personnes à la recherche d'un emploi ; il n'y a alors pas de contradiction à utiliser à la fois des instruments de contrôle et des instruments de réinsertion.

\section{Une recentralisation relative de la mise en œuvre de la loi}

Les politiques suisses pour l'emploi du milieu des années 80 étaient avant tout des politiques d'indemnisation, mises en œuvre par les communes. Ce système avait laissé se développer de fortes disparités dans l'accompagnement des chômeurs. Pour une part, les grandes communes du pays - Zürich,
Genève, Bâle, Lausanne, etc. - avaient pu recruter du personnel qualifié capable de mobiliser une expertise sur le marché local du travail et avaient pu financer des mesures actives pour l'emploi. Pour le reste, l'immense majorité des communes suisses s'étaient contentées de faire "pointer» les chômeurs et de leur verser les indemnités.

La loi de 1995 sur l'emploi organise la cantonalisation des politiques de l'emploi. Les cantons ont ainsi la charge de l'organisation du service public de l'emploi. Le législateur fédéral fixe des objectifs, des procédures, des formes administratives, impose l'usage d'instruments d'action publique déterminés, mais confie l'exécution des politiques aux cantons. La cantonalisation a pour but premier d'homogénéiser les capacités d'intervention publique sur l'ensemble du territoire fédéral. Le législateur fédéral a également imposé de nombreuses procédures de contrôle de la mise en œuvre de la loi par les cantons.

Au-delà du bréviaire du fédéralisme d'exécution ${ }^{5}$ "l'arène confédérale décide, l'arène cantonale met en æuvre »-, la loi de 95 introduit une série d'innovations administratives qui brouillent en partie le mécanisme.

Tout d'abord, les modalités d'organisation du service public de l'emploi sont fixées dans la loi. Les cantons ont mis en place des offices régionaux de placement (ORP), structurés au plan régional ou sectoriel, indépendants les uns des autres. Cette organisation à un niveau administratif « régional », qui ne correspond à rien dans le système institutionnel helvétique ${ }^{6}$, place le niveau opérationnel de mise en œuvre de la loi dans un double rapport d'autorité. D'une part, les ORP dépendent directement des administrations cantonales du travail, rattachées le plus souvent au département de l'économie. D'autre part, les ORP sont liés au niveau fédéral, en relation avec le SECO, dans le cadre de la filière du service public de l'emploi.

\footnotetext{
${ }^{5}$ La Confédération n'a pas d'administration à disposition dans les cantons. La poste, les Chemins de fer ou l'administration du gouvernement central sont les seules dont dispose la Confédération. Il n'y a en Suisse ni police fédérale, ni services fiscaux fédéraux dans les cantons, ni représentations dans les cantons de l'administration de l'économie.

${ }^{6}$ Le fédéralisme suisse est structuré par les trois niveaux très autonomes les uns par rapport aux autres que sont la Confédération, les cantons et les communes.
} 
Enfin, la loi associe au pilotage de la mise en œuvre une instance tripartite, incluant les employeurs et les salariés et qui ne joue ici qu'un rôle consultatif. Cette commission a pour vocation d'apporter au processus opérationnel une expertise du marché cantonal du travail qui permette de fonder les orientations retenues dans le canton quant à la mise en œuvre des instruments de la loi. Cette expertise est particulièrement attendue dans le domaine de la formation professionnelle. De nouveau, cette commission tripartite a une vocation technique. Il ne s'agit pas pour elle d'organiser une représentation d'intérêts qui pourrait ressembler à une forme régionale de concertation sociale ou de dialogue social territorialisé.

Ainsi, la cantonalisation des politiques de l'emploi est limitée par le poids de l'influence de la Confédération - liens avec les ORP, directives, contrôle de mise en œuvre, etc. -, par celui des commissions tripartites qui représentent employeurs et salariés, mais elle l'est aussi par les habitudes prises par les grandes communes du pays - Zürich, Genève, Bâle,
Lugano, Lausanne, etc. - qui n'ont souvent pas renoncé à leurs propres dispositifs. Entre homogénéité et différenciation se construit ainsi un jeu complexe de forces contradictoires. Les traditions politiques différenciées desquelles procèdent les cantons ou encore leurs choix politiques renforcent l'hétérogénéité en ce domaine.

\section{DES MODES DISPARATES DE MISE EN OEUVRE DE LA LOI}

Le fédéralisme d'exécution helvétique représente un point commun essentiel avec l'organisation politique et administrative de l'Union européenne. Dans un tel contexte institutionnel, la conformité de l'application des lois par les cantons avec l'esprit, les finalités, mais aussi avec le fonctionnement des instruments inscrits dans les textes fédéraux fait l'objet d'une grande incertitude. La mise en œuvre de la loi sur

\section{Encadré 1 \\ Une recherche sur l'analyse des modes de mise en œuvre, par les cantons, de la loi fédérale sur le chômage}

La recherche financée par le Programme national de recherche du Fonds national de la recherche suisse (Berne), conduite au sein de l'Institut d'études politiques et internationales de l'université de Lausanne, était structurée en deux étapes essentielles:

$1 /$ Analyse des modes de mise en œuvre cantonale de la loi au moyen de statistiques mises à disposition par le Secrétariat d'État à l'Économie (SECO) et d'une enquête par questionnaire auprès des 26 administrations cantonales de l'emploi. Les indicateurs présentés dans l'encadré 2 constituent l'objet principal de cette phase d'enquête. Ils permettent de mesurer l'utilisation, par les différents cantons, des instruments de mise en œuvre mis à disposition par le texte de loi fédéral. Ces instruments sont ici classés entre instruments de réinsertion et instruments de contrôle/sanction des chômeurs.

2/Analyse qualitative des logiques de mise en œuvre de la loi dans sept cantons suisses : Genève, Fribourg, Bâle-ville, Berne, le Tessin, Obwald et Nidwald. II s'agit, à ce stade, de tenter d'expliquer les disparités dans les modes de mise en œuvre au moyen d'enquêtes qualitatives au sein de l'administration cantonale de l'emploi, des Offices régionaux de placement, mais aussi du réseau de pilotage et de mise en œuvre de la loi dans les cantons. Ce réseau inclut, outre les instances politiques et administratives, les associations patronales, syndicales du canton ou encore les associations de chômeurs ou des entreprises proposant des formations. Les méthodes de l'analyse qualitative des réseaux ont été utilisées : analyse de la répartition des sièges dans les commissions tripartites et autres, des positions dans les documents officiels, entretiens croisés vérifiant l'intensité des contacts, la conformité des positions et réalisation d'une vingtaine d'entretiens (par étude de cas) d'une à deux heures portant sur le déroulement concret de l'activité ainsi que sur les contacts et coopérations des personnes interrogées. 


\section{Tableau 1}

Modes de mise en œurre de la loi sur l'emploi par les cantons

Réinsertion

Elevée

Basse

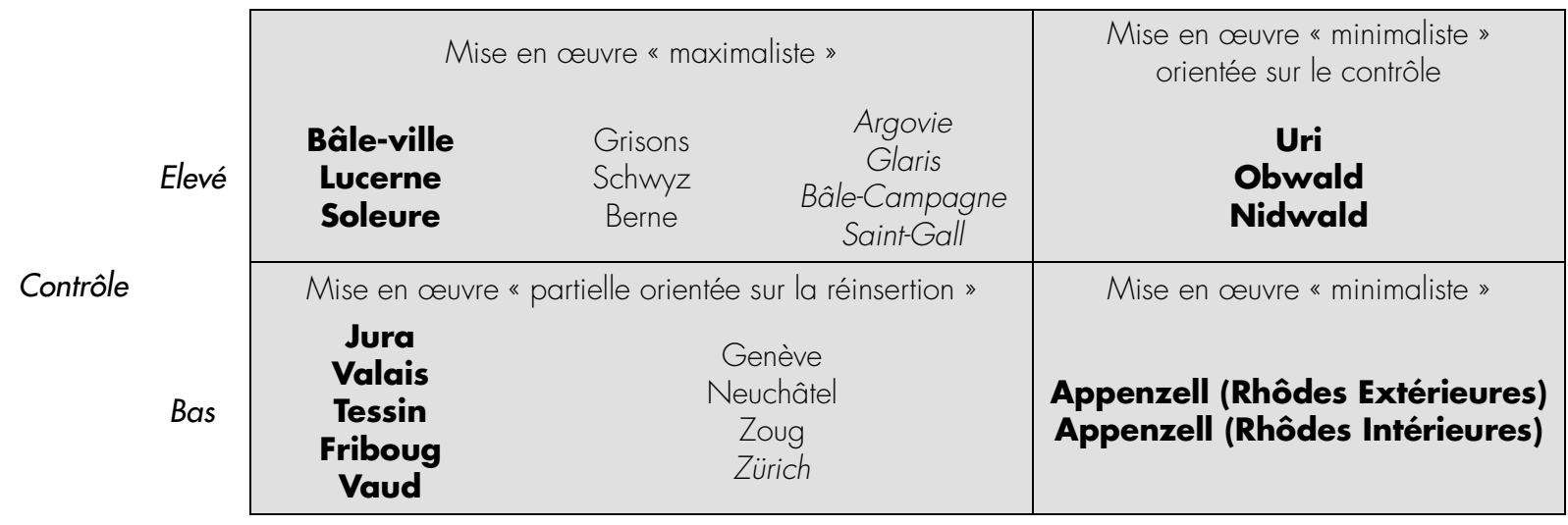

Note de lecture : Les cantons dont le nom est en caractères gras se situent au-dessus de la moyenne fédérale pour la totalité des indicateurs de réinsertion. Les cantons dont le nom est en caractères ordinaires se situent au-dessus de la moyenne fédérale pour les deux tiers de ces indicateurs. Les cantons dont le nom est en caractères italiques ne présentent des taux au-dessus de la moyenne fédérale que pour un seul indicateur de réinsertion. Le classement de ce dernier groupe est ainsi relativement peu significatif. Seuls 5 cantons parmi les 24 répondants se trouvent dans cette situation.

Source : auteur, sur la base des indicateurs mis à disposition par les services du secrétariat d'État à l'Économie.

l'indemnisation et la lutte contre le chômage par les cantons suisses est particulièrement intéressante à étudier, à cet égard, dans la mesure où elle juxtapose des instruments différents, voire contradictoires, pour atteindre un même objectif. Mesures de réinsertion et de contrôle des chômeurs sont ainsi mobilisées afin de réduire le chômage en favorisant leur réintégration sur le marché du travail.

\section{Mesurer les disparités}

Quelques années seulement après la première phase de mise en application de la nouvelle loi sur l'assurance-chômage, les premiers rapports d'évaluation disponibles ont montré que les différents cantons suisses procédaient à des mises en œuvre disparates de ce texte fédéral, pourtant destiné à homogénéiser les pratiques dans le domaine des politiques de l'emploi (Curti, Meins, 1999). Ce constat a été corroboré par une recherche ${ }^{7}$ qui a testé la mise en œuvre, par les cantons, des deux principaux types d'instruments mis à disposition par la loi : la réinsertion et le contrôle.

${ }^{7} C f$. note 1 et encadré 1.
Afin de tester la façon dont les ORP ont utilisé les différents instruments de politiques de l'emploi mis à disposition par le législateur fédéral, des indicateurs d'usage des mesures de contrôle et de réinsertion ont été construits (encadré 2) et appliqués à la quasitotalité des cantons suisses.

En fonction des indicateurs mentionnés dans l'encadré 1, il a été possible d'élaborer la classification suivante (tableau 1) des cantons selon le mode de mise en œuvre de la loi fédérale pour l'emploi.

La lecture du tableau 1 indique que trois usages de la loi fédérale pour l'emploi peuvent être distingués en fonction de l'intensité du recours aux différents instruments des politiques de l'emploi disponibles dans la loi. Le premier usage est celui des cantons, qui appliquent la loi en conformité avec les intentions du législateur fédéral et selon les recommandations du SECO. Ces cantons font un usage important et conjoint des mesures de réinsertion, parmi lesquelles on compte des mesures de formation, mais aussi des mesures de contrôle des chômeurs. Ce groupe est avant tout constitué par les cantons de Suisse alémanique et notamment de la Suisse du Nord et de l'Est. Le deuxième usage consiste à utiliser principalement les mesures de réinsertion et à négliger les mesures 


\section{Encadré 2 \\ Indicateurs de réinsertion et de contrôle dans la mise en œuvre de la loi}

Indicateurs de réinsertion

a) Le niveau de développement de la logistique des mesures de marché du travail (LMMT). Pour mettre en œuvre les politiques de l'emploi, les cantons ont la possibilité d'instituer une logistique des mesures de marché du travail. Ce service mobilise l'expertise compétente et élabore le contenu des mesures. Un bon niveau de développement de ce service indique un fort investissement du canton en faveur de la réinsertion.

b) La présence/absence d'essais pilotes ou mesures cantonales spécifiques. La loi (Art. 110 a) autorise les cantons à effectuer des essais pilotes de durée limitée dérogeant à la loi. De tels essais pilotes ou mesures cantonales spécifiques attestent d'un fort engagement du canton dans la lutte contre le chômage.

c) Le taux de réalisation des mesures actives pour l'emploi demandé par la Confédération aux cantons. Plus un canton réalise des mesures actives du marché du travail, plus sa mise en œuvre de la loi sur l'emploi s'inscrit dans une politique de réinsertion active.

Indicateurs de contrôle

Le nombre de sanctions décidées par le service public de l'emploi par demandeur d'emploi. Plus le nombre de sanctions est élevé, plus le canton inscrit sa mise en œuvre dans la dimension contrôle.

Les indicateurs a et $b$ ont été obtenus au moyen d'une enquête par questionnaire, directement adressée aux administrations cantonales de l'emploi (24 cantons suisses sur les 26 ont répondu à l'enquête). L'indicateur c ainsi que l'indicateur de contrôle ont été mis à disposition directement par l'administration fédérale (Secrétariat à l'Économie).

de contrôle des chômeurs. On trouve dans ce groupe l'ensemble des cantons de Suisse latine - francophone et italophone. Enfin, le troisième usage est qualifié de «minimaliste» au sens où les cantons concernés soit n'utilisent pas ou très peu l'ensemble des instruments contenus dans la loi, soit utilisent seulement les instruments de contrôle des chômeurs8. Il s'agit des cantons alémaniques de Suisse centrale.

Différents facteurs permettent d'expliquer ces disparités inter-cantonales dans les modalités de mise en œuvre de la loi. En premier lieu, il existe une certaine correspondance entre l'intensité du chômage et le mode de mise en œuvre de la loi : les cantons qui présentent un faible taux de chômage - inférieur à $2 \%{ }^{9}$ - sont ceux qui procèdent à une utilisation minimaliste de la loi ou qui ne recourent qu'aux instruments de contrôle. En revanche, les cantons qui

\footnotetext{
${ }^{8}$ En raison du petit nombre de cantons concernés, nous renonçons ici à distinguer entre les deux usages de la loi soulignés ici.

${ }^{9}$ L'administration fédérale de l'économie (SECO) fournit des données actualisées - mais aussi une série depuis le début des années 90 - sur les taux de chômage cantonaux (http://ams.jobarea.ch/f_home. asp.)
}

connaissent les taux de chômage les plus élevés, les cantons de Suisse latine, sont aussi ceux qui recourent le plus volontiers aux instruments de réinsertion, et notamment aux mesures de formation professionnelle. Cependant, l'intensité du chômage n'explique pas la totalité de la distribution des cantons. Des cantons dont les marchés du travail sont assez similaires - Berne et Fribourg par exemple ou encore Schwyz et $\mathrm{Ob}$ - et Nidwald - adoptent des modes de mise en œuvre de la loi différenciés. Plus encore, l'intensité du problème du chômage peut expliquer l'intensité dans l'utilisation des différents instruments, mais elle ne peut pas expliquer la préférence des cantons pour des instruments de contrôle ou au contraire, pour des instruments de réinsertion.

Les rapports de force et les modes d'interaction qui s'établissent au sein des réseaux cantonaux de pilotage et de mise en œuvre de la loi sur le chômage doivent aussi être pris en compte. Nos études de cas conduites dans six cantons suisses (encadré 1) ont ciblé cette dimension à travers l'analyse de l'organe tripartite représentants patronaux, salariés et du service public 
de l'emploi - de pilotage de la mise en œuvre de la loi mais aussi des réseaux moins formels d'interconnaissance (Battaglini, Giraud, 2004). Ces réseaux fonctionnent de façon plus ou moins équilibrée dans les différents cantons suisses. Les analyses conduites dans sept cantons - Genève, Fribourg, Bâle-ville, Tessin, Nidwald, Obwald et Berne - ont permis d'isoler trois principaux types de réseaux de pilotage et de mise en œuvre. Chacun de ces types de réseaux correspond précisément à un mode de mise en œuvre de la loi :

\section{Une mise en œuvre minimaliste orientée sur le contrôle des chômeurs}

En Suisse centrale - par exemple dans les cantons d'Obwald et de Nidwald - les instances de consultation des partenaires sociaux ne fonctionnent pratiquement pas. Dans ces cantons fortement dominés par une culture politique libérale et conservatrice, il n'existe aucune section syndicale organisée. Des représentants syndicaux venus de cantons voisins, dotés d'une culture industrielle plus forte, tentent parfois de représenter les intérêts salariés dans des commissions officielles, mais cette représentation est irrégulière et peu efficace. Les réseaux de pouvoir pertinents dans ces régions sont ceux qui lient les organisations patronales aux administrations, notamment par le biais des partis politiques bourgeois. Le mode de mise en œuvre de la loi observé dans ces régions correspond à une mise en œuvre partielle orientée sur le contrôle des chômeurs.

\section{La primauté aux instruments de réinsertion}

À Genève et au Tessin et, dans une moindre mesure à Fribourg, les syndicats sont installés dans des positions fortes qui leur permettent de négocier avec le pouvoir politique cantonal la quasi-totalité des mesures d'action publique qui ont des conséquences dans le domaine économique et social (Battaglini, Giraud, 2003, pp. 300 et s.). Dans ces cantons, les syndicats bénéficient non seulement de fortes traditions de consultation de leur avis au moment de la prise de décision politique, mais ils disposent également de réseaux politiques - élus au parlement cantonal, fonctionnaires de haut niveau, etc. - qui leur permettent de défendre les intérêts des salariés de façon efficace. Face à cette représentation forte des intérêts du salariat, les organisations patronales et les partis bourgeois ne sont pas démunis et disposent également d'un accès aux organes officiels de consultation comme aux réseaux politiques et administratifs. Le mode de mise en œuvre relevé dans ces trois cantons privilégie les instruments de réinsertion.

\section{Une utilisation intensive des instruments de réinsertion et de contrôle}

À Berne et à Bâle-ville, les réseaux de mise en œuvre et de pilotage de la loi sur le chômage sont fortement centrés sur l'administration. Les instances patronales et syndicales, mais aussi les associations de chômeurs ou plus largement le milieu associatif de l'insertion, sont marginalisés. À la différence de ce que l'on peut constater dans le premier type de réseaux de pilotage, il n'y a pas ici une véritable appropriation des réseaux de pilotage et de mise en œuvre par un type précis d'organisation d'intérêts, mais plutôt un centrage du pilotage de la mise en œuvre sur les seuls réseaux administratifs opérationnels. La loi fédérale sur le chômage visait précisément à introduire une relation privilégiée entre les instances opérationnelles de mise en œuvre de la loi (les ORP) et le SECO, instance politique de niveau fédéral. Ce pilotage administratif, outre le fait qu'il constitue une tentative de détournement de l'influence politique des cantons, a permis à certains d'entre eux - Bâle-ville ou Berne par exemple - d'abandonner pratiquement la gestion des politiques pour l'emploi à des administrations cantonales pilotées par la Confédération, dans le cadre d'une relation presque directe entre services administratifs fédéraux et cantonaux. La mise en œuvre relevée ici est maximaliste au sens où les instruments de réinsertion comme les instruments de contrôle font l'objet d'une utilisation intensive.

\section{LA FORMATION : DIVERGENCES D'INTERPRÉTATION ET RÉSEAUX DE POUVOIR}

Derrière ces groupes de cantons isolés sur la base d'indicateurs chiffrés réducteurs, mais qui permettent de tenir compte de l'ensemble des cantons suisses, se 
profilent des pratiques concrètes de traitement des chômeurs qui correspondent à des formes bien identifiées d'interactions entre l'État et la société civile. Plus encore, ces divergences dans les pratiques renvoient à des conceptions du chômage, de ses causes, des moyens de le traiter, en bref, à des interprétations du chômage et des politiques de l'emploi qui sont également divergentes.

Ces divergences d'interprétation sont particulièrement apparentes en matière de sanction des chômeurs. Alors même que l'instance fédérale de coordination des politiques de l'emploi - le SECO édicte des recommandations précises sur les motifs et les niveaux des sanctions à prononcer ${ }^{10}$, la grande variabilité des taux de sanctions prononcées par les ORP indique que les cantons interprètent de façons assez diverses les motifs officiellement reconnus. Certaines administrations cantonales prononcent effectivement des sanctions pour la totalité des cas envisagés, alors que d'autres ne prononcent des sanctions que dans les cas les plus extrêmes (Battaglini, Giraud, 2005). Ainsi, il est possible de sanctionner des chômeurs en raison de leur comportement sur le marché du travail - absence de recherche active d'emploi pendant la période du préavis de licenciement ou refus d'un «emploi convenable» au terme de la loi. Mais il est également possible de sanctionner les chômeurs en raison d'un comportement jugé inadéquat vis-à-vis de l'administration de l'emploi elle-même - non présentation à un rendezvous, refus de suivre ou suivi irrégulier d'une mesure de formation.

\section{La formation comme enjeu-clé d'interprétation}

La formation est également un enjeu-clé pour illustrer divergences et conflits d'interprétation. La formation peut en effet être utilisée, conformément aux textes, pour mobiliser, stimuler ou reconvertir des personnes en situation de chômage. Mais la formation peut aussi faire l'objet d'un conflit entre le demandeur d'emploi et le conseiller-placeur. Elle peut alors devenir une

${ }^{10}$ Il existe ainsi différentes «grilles » (Raster) produites par le SECO et diffusées dans l'ensemble des ORP permettant de mettre en regard les incartades à la loi sur le chômage et le niveau des sanctions à prononcer. mise à l'épreuve des personnes à la recherche d'un emploi ou peut servir à imposer des reconversions non souhaitées par les individus. Le refus d'une formation pouvant donner lieu à des sanctions, une relation de pouvoir univoque s'établit alors entre le conseillerplaceur et le chômeur.

Nos études de cas ont mis en évidence des disparités importantes dans les structures des réseaux de pilotage et de mise en œuvre de la loi. Des usages et des interprétations spécifiques de la formation professionnelle peuvent être attachés à ces différents types de réseaux.

Les catégories développées par Jon Elster (1992) dans son analyse de la répartition des ressources rares saisies dans une tension entre principes de justice globaux et locaux semblent pouvoir s'appliquer de façon utile aux politiques de l'emploi (Gazier, 2000). Le «besoin », «l'efficacité » et le "mérite» sont constitués politiquement mais sont spontanément mobilisés par les conseillers-placeurs pour motiver les attributions de formations. Ces critères s'appliquent d'ailleurs à la fois aux individus et à une conception du bien commun, d'un intérêt général territorialisé. Entre principe de justice global, principe de justice local, intérêt de l'individu et intérêt général, les possibilités pour les conseillersplaceurs de justifier leurs décisions sont multiples. Ces différentes décisions correspondent d'ailleurs à des ordres de motivation différents (Lindenberg et al., 2000). Elles ne constituent pas toujours des décisions individuelles mais sont encadrées par une série de procédures, contrôles collectifs, et aussi de «sens commun » pour reprendre l'expression de Elster (1992), de sens partagé, dominant, dont la valeur et la validité sont ancrées dans des territoires.

Ces «sens communs», ces interprétations et discours qui dominent localement constituent l'objet des analyses qui suivent. Les interprétations du rôle, de l'efficacité, de la place des mesures de formation - conception et mise en œuvre - dans l'ensemble du dispositif suisse des politiques de l'emploi sont étudiées sur la base d'entretiens ${ }^{11}$ approfondis conduits à différents niveaux de l'administration de l'emploi et ce dans les sept cantons analysés.

${ }^{11}$ Six par canton en moyenne. 
Membres syndicalistes ou représentants patronaux des commissions triparties d'orientation des ORP, responsables cantonaux du travail, de l'économie, directeurs ou directrices d'ORP, conseillers-placeurs, représentants d'associations de chômeurs comptent parmi les personnes dont les discours sont analysés ici de façon synthétique.

Dans certains contextes cantonaux, des clivages importants existent. Les conflits d'interprétation ne se déploient ainsi pas seulement au sein de l'arène fédérale entre la Confédération et les cantons ou entre les cantons entre eux. Ces conflits peuvent traverser les réseaux d'action publique. Au-delà des oppositions s'impose cependant un discours dominant, un «sens commun », celui qui se comprend tout naturellement, qu'il n'est guère nécessaire d'expliquer ou de justifier. Ces images, ces sens communs et partagés, particulièrement disparates d'un canton à l'autre, sont ceux que nous allons avant tout mettre en valeur ici.

\section{La formation professionnelle comme réponse à un besoin non contesté}

Bâle-ville et Genève sont les deux seuls cantons parmi nos sept études de cas à disposer d'une tradition ancienne en matière de formation comme politique de l'emploi. Pour des raisons économiques (centres économiques et urbains importants) et politiques (tradition syndicale et de gauche politique forte), les deux grandes villes-États de Suisse proposent aux chômeurs des accompagnements dans le domaine de la formation professionnelle depuis plusieurs décennies déjà.

Dans ces deux cantons, les expériences accumulées au fil des années permettent aux administrations cantonales de l'emploi de constituer une expertise précieuse, notamment pour l'élaboration des mesures de formation. Des services spécialisés élaborent des catalogues de formation détaillées et différenciées (formations collectives, individuelles de coaching, avec suivi individualisé, etc.) ayant des objectifs divers : mobilisation, aptitude au placement, langue, perfectionnement professionnel, etc.

Dans ces contextes cantonaux, les organisations représentatives des partenaires sociaux sont souvent également des prestataires de formation et soutiennent largement les politiques conduites dans le cadre de la mise en œuvre de la loi sur le chômage. Par ailleurs, un marché cantonal actif et diversifié de la formation permet une mise en concurrence de prestataires de différents types. Les écoles professionnelles publiques, les organisations collectives comme les syndicats ou les chambres consulaires, mais aussi de nombreux prestataires privés alimentent l'offre cantonale.

Entre la puissance d'évocation de la tradition sociale et le haut niveau d'investissement du tissu social, les interprétations de la formation professionnelle relevées sur le terrain sont largement positives parmi les conseillers-placeurs en charge de la mise en œuvre opérationnelle. Dans les deux cas, la question de l'efficacité des mesures est peu interrogée ; la formation professionnelle fait l'objet d'un assez large consensus entre acteurs et répond pour tous à un « besoin » de la part des chômeurs.

Au-delà de ces similarités, les usages bâlois et genevois de la formation acceptent malgré tout un certain nombre de différences. À Bâle-ville, les usages de la formation sont avant tout centrés sur une évaluation des cas individuels. À Genève, les formations sont souvent, comme les autres mesures actives, attribuées à des chômeurs en fin de droit de façon à permettre à ces derniers de proroger leurs droits à une période d'indemnisation de près de deux années $^{12}$. L'interprétation de la formation qui s'impose ainsi à Genève est clairement centrée sur la notion de traitement social du chômage.

\section{La formation : une approche centrée sur le mérite}

Dans le cas des cantons de Nid- et Obwald, les représentants politiques ou patronaux, mais aussi les acteurs administratifs cantonaux jusqu'aux conseillersplaceurs des ORP affichent une franche hostilité et au mieux un fort scepticisme à l'égard des mesures de formation. Condamnées pour leur inefficacité, les politiques de formation font l'objet d'une interprétation restrictive. Les acteurs cantonaux de la politique de l'emploi estiment que l'ajustement des compétences des chômeurs aux besoins du marché du travail ne fait pas partie des attributions des politiques de

\footnotetext{
${ }^{12}$ Le canton prolonge ainsi l'intervention fédérale alors que l'aide sociale fait intervenir les finances des communes du canton.
} 
l'emploi. Le service public de l'emploi «ne doit pas être considéré comme un service gratuit » (Entretien ORP communes Nidwald et Obwald) à disposition des chômeurs.

Selon l'interprétation dominante dans ces cantons, les chômeurs doivent eux-mêmes trouver les moyens de s'adapter aux contours du marché du travail en acceptant les postes disponibles sur le marché du travail. La responsabilité des individus face à leur situation est centrale dans les discours recueillis. Cette interprétation s'appuie sur des expertises disponibles qui démontreraient l'inefficacité des politiques de formation professionnelle.

Les mesures de formation ainsi majoritairement interprétées comme des privilèges inefficaces sont écartées par les conseillers placeurs qui ne les utilisent que rarement.

Dans le cas de ces cantons à la culture politique conservatrice et libérale, les discours sur la formation n'engendrent pas de véritables conflits d'interprétation dans le contexte régional. La faiblesse structurelle des forces traditionnellement orientées à gauche - partis, élus - et l'absence d'organisations syndicales expliquent le caractère univoque des perceptions de la formation. En revanche, l'incompréhension règne quant aux directives fédérales qui font l'apologie des mesures de formation.

\section{La formation : quelle efficacité individuelle et collective?}

Enfin, dans certains cantons «maximalistes» (Berne), les plus orthodoxes par rapport aux intentions de la loi, mais aussi dans certains cantons « centrés sur la réinsertion » (Fribourg et le Tessin), la question de l'efficacité des politiques de formation face au chômage fait l'objet d'un débat ouvert entre défenseurs et sceptiques. Les interprétations - et leurs justifications - relèvent, dans ces contextes cantonaux, de débats en partie pragmatiques mais aussi de nature moraliste; l'interrogation ne porte alors pas tant sur le mérite, comme dans le cas précédent, que sur l'efficacité, pour reprendre les termes de Elster.

Au moment de notre enquête dans la première moitié des années 2000, la situation du marché du travail suisse s'était améliorée. Cependant, une forte propor- tion de chômeurs était difficile à réinsérer. Contrairement à d'autres types de cantons, les conseillersplaceurs faisaient un usage circonstancié des mesures de formation, sans les associer systématiquement à des privilèges indus ni à une tradition constitutive de l'identité des politiques de l'emploi dans le canton et que l'on n'interroge guère. Dans ce dernier groupe de cantons, on retrouve d'une part des interprétations pragmatiques, fondées sur une expertise argumentée des dossiers ; d'autre part, des interprétations moralistes qui mettent en doute non seulement l'efficacité technique des mesures de formation, mais questionnent également le «mérite », la valeur morale des chômeurs face à une mesure de formation perçue comme un investissement coûteux pour l'administration de l'emploi, donc pour la collectivité.

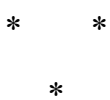

Le cas des politiques de l'emploi en Suisse constitue une illustration éclairante de la complexité des interactions multiniveaux qui caractérisent, aujourd'hui, la plupart des régulations de politiques publiques contemporaines, comme dans les pays de l'Union européenne. Cette complexité, fondée sur différents types de discontinuités dans le régime de mise en œuvre des politiques publiques, permet une véritable pluralité de résultats. Cette pluralité est elle-même liée à une diversité des perceptions, des débats, des équilibres politiques qui fondent ces mises en œuvre.

Tout d'abord, les programmes d'action publique sont de plus en plus souvent, en Suisse comme ailleurs, composés d'une grande variété de mesures. Il s'agit alors parfois de combinatoires d'instruments d'action publique (policy mix) fondées sur l'idée que seule une pluralité d'actions peut résoudre efficacement les problèmes en cause. Dans d'autres cas, ces « collages » entre des objectifs stratégiques et des instruments opérationnels, pas toujours cohérents entre eux, résultent plutôt de compromis politiques. Des coalitions politiques, politiquement complexes, comme on les trouve en Suisse ou dans l'Union européenne, débouchent souvent sur de telles stratégies différenciées. Ces juxtapositions d'instruments et de mesures occasionnent potentiellement des assemblages d'actions fort divers au stade de la mise en œuvre. 
Dans le cas des politiques suisses de l'emploi, on observe la juxtaposition d'instruments de contrôle et de réinsertion ouvrant un espace important à de possibles divergences et conflits d'interprétation de la loi au stade de la mise en œuvre. Le chômeur est ainsi tour à tour présenté comme une personne faisant face à un marché du travail exigeant et qui doit ainsi légitimement être aidée par le service public de l'emploi. Mais il est également considéré comme une personne tentée de commettre des abus sur le marché du travail et devant être soumise à un contrôle étroit de son comportement à la fois sur le marché du travail et dans sa relation au service public de l'emploi.

Ensuite, la mise en œuvre de la loi se décline selon une succession de délégations à des niveaux institutionnels inférieurs ; dans notre cas, de la Confédération vers les cantons, mais aussi en direction des Offices régionaux de placement, instance opérationnelle de mise en œuvre de la loi. En dépit de l'impact des instruments purement administratifs de contrôle de la mise en œuvre de la loi, les cantons, mais aussi les réseaux politiques et associatifs du domaine dans le contexte cantonal, exercent une influence particulièrement importante sur l'orientation de la mise en œuvre des politiques publiques.

Précisément, dans le cadre de ce contexte opérationnel de mise en œuvre, les conflits d'interprétation de la loi peuvent surgir et même s'appliquer à des dispositifs d'action publique comme les instruments de formation. De Genève à Nidwald, la formation passe du statut de dispositif consensuel de l'insertion économique et de soutien de la situation sociale des chômeurs au statut de mesure illégitime au plan de l'action publique et inefficace au plan économique. La formation, vue en Suisse occidentale comme un instrument indispensable de soutien aux chômeurs, se transforme, dans certaines régions de Suisse orientale, en un dispositif trop coûteux et immérité par les personnes à la recherche d'un emploi.

Les arènes cantonales constituent des scènes publiques autonomes dans lesquelles les acteurs-clés - politiques, administratifs, syndicaux, patronaux, associatifs, etc. - de la mise en œuvre des politiques publiques forment des réseaux de pouvoir qui structurent largement l'interprétation des instruments dominants des politiques publiques. Cette phase d'interprétation joue un rôle particulièrement important pour comprendre comment les acteurs administratifs en charge de la mise en œuvre de terrain (street-level bureaucrats) se saisissent de ces instruments dans le cadre de leur activité de soutien au placement ou de contrôle des chômeurs. Ainsi en Suisse, comme dans d'autres pays, les dispositifs publics de formation donnent lieu à des usages particulièrement contrastés. On ne peut comprendre ces usages en dehors d'une analyse approfondie des réseaux de pouvoir locaux desquels ils émergent ou encore des discours, des sens partagés ou des conflits d'interprétation - qui animent ces réseaux de pouvoir.

\section{Bibliographie}

Barbier J.-C. (2004), « Une européanisation des politiques de l'emploi ? Réflexions d'étape à propos de la stratégie coordonnée pour l'emploi », Travail et Emploi, ${ }^{\circ} 100$, octobre, pp. 11-26.

Barbier J.-C. (2005), « The European Employment Strategy: A channel for activating social protection?» in Zeitlin J. and Pochet P. with Magnusson L. (Eds.), The Open Method of Coordination in Action: The
European Employment and Social Inclusion Strategies, Brussels: PIE-Peter Lang, pp. 417-446.

Battaglini M., Giraud O (2003), « Policy Styles and the Swiss Executive Federalism: Comparing diverging styles of cantonal implementation of the federal law on unemployment», Swiss Political Science Review 9, (1 Sonderheft « Schweizer Föderalismus in vergleichender Perspektive »), pp. 285-308. 
Battaglini M., Giraud O. (2004), « Mise en œuvre des politiques pour l'emploi et pouvoir régional : le poids des réseaux sociaux et politiques », Revue Suisse de Sociologie, Vol. 30, n 3, pp. 363-379.

Bel M., Méhaut Ph., Mériaux O (dir.), (2003), La décentralisation de la formation professionnelle Quels changements dans la conduite de l'action publique ?, Paris, L'Harmattan.

Berthet $\mathrm{T}$ (dir.) (2005), Des emplois près de chez vous? La territorialisation des politiques d'emploi en questions, Bordeaux, Presses Universitaires de Bordeaux.

Bonoli G., Mach A. (2001), "The New Swiss Employment Puzzle », Revue suisse de science politique 7 (2), pp. 81-94.

Curti M., Meins E. (1999), «Politiques suisse du marché du travail - Différences résultant de sa mise en application au niveau du service de l'emploi », $L a$ Vie Économique (2/99), pp. 64-70.

Elster J. (1992), Local Justice. How Insitutions Allocate Scarce Goods and Necessary Burdens, Cambridge, Cambridge University Press.

Gazier B. (2000), «L'articulation justice locale/ justice globale : le cas des marchés transitionnels du travail », Revue Économique, vol. 51, n 3, pp. 571581.

Giraud O., Battaglini M. (2005), « Pilotage et mise en œuvre des politiques pour l'emploi en Suisse : analyse de la régulation de l'emploi entre stratégie fédérale et besoins territorialisés », Synthèse 26, PNR 43. Fonds National Suisse, Berne (http://www.nfp43.unibe.ch/ $\mathrm{PDF} /$ synthesis26.pdf).

Giraud O., Braun D. (dir.) (1999), « Les politiques de l'emploi en Suisse à la fin des années 90 : modèle(s) en transition », Lausanne: Numéro spécial Revue Économique et Sociale.

Giriens P.-Y., Staufer J. (1999), « Deuxième révision de la loi sur l'assurance-chômage: genèse d'un compromis ", Mach A. (dir.), Globalisation, néo- libéralisme et politiques publiques dans la Suisse des années 90, Zürich : Seismo, pp. 105-143.

Heikkilä M. (1999), «A brief introdution to the topic », in O'Conghaile, W. (Ed.), Linking welfare and work, Dublin, European Foundation for the Improvement of Living and Work Conditions, pp. 1-5 and 87-91.

Jobert B. (1998), « La régulation politique - le point de vue d'un politiste », Commaille J., Jobert B. (dir.), Les métamorphoses de la régulation politique, Paris, LGDJ, pp. 119-144.

Knoepfel P., Larrue C., Varone F. (2006), Analyse et pilotage des politiques publiques, Zürich, Coire, Ruegger Veralag.

Lindenberg, S. (2000), «The extension of rationality : Framing versus cognitive rationality » in Baechler J., Chazel F. and Kamrane R. (Eds.), L'Acteur et ses Raisons. Mélanges en l'honneur de Raymond Boudon, pp. 168-204.

Magnin Ch. (2004), « Beratung und Kontrolle. Ein für den aktivierenden Staat typisches Handlungsdilemma », Revue suisse de sociologie 30, pp. 339-361.

OCDE (1996), Politiques du marché du travail en Suisse, Paris, OCDE.

OFS - Office Fédéral de la Statistique - (2005), (http://www.bfs.admin.ch/bfs/portal/fr/index/themen /arbeit_und_e/uebersicht/blank/panorama/erwerbsbeteiligung. html).

Pressman J.L., Wildavsky A. (1973), Implementation, Berkeley, University of California Press.

Richardson J. et al. (1982), « The concept of policy style» in Richardson J. (dir.), Policy Styles in Western Europe, Londres, Allen \& Unwin.

Schmidt M. G. (1995), «Vollbeschäftigung und Arbeitslosigkeit in der Schweiz. Vom Sonderweg zum Normalfall », Politische Vierteljahresschrift 36 (1), pp. 35-48.

SECO (2006), Circulaire relative aux mesures $d u$ marché du travail (MMT), Direction du Travail, Berne. 
Svensson C. (2001), Swedish Social Democracy \& the Third Way - a delicate affair. Multiple Third Ways : European Social Democracy facing the Twin Revolution of Globalisation and Knowledge Society, Cuperus R., Duffek K. and Kandel J., Amsterdam, Colofon, pp. 219-226.

Sylla S.N., Barbier J.-C (2006), Une synthèse de la littérature à propos de l'activation. Analyse compa- rative de l'activation de la protection sociale en France, Grande-Bretagne, Allemagne et Danemark, dans le cadre des lignes directrices de la stratégie européenne pour l'emploi, Rapport de recherche pour la DARES, ministère du travail. (http://matisse.univparis1.fr/barbier/perso/CEE-RF2005.pdf)

De Terssac G. (2003), La théorie de la régulation sociale de Jean-Daniel Reynaud, Paris, La Découverte.

\section{Résumé}

\section{La formation \\ comme politique d'activation des chômeurs en Suisse : divergences $d$ 'interprétation \\ Olivier Giraud}

Cet article propose une analyse des divergences d'interprétation des politiques d'activation des chômeurs à l'aune des discours et des usages des politiques de formation développées dans le contexte du fédéralisme suisse. Le caractère polysémique de la notion d'activation attachée aux politiques de la formation et de l'emploi rend possible des conflits d'interprétation autour du rôle de ces mesures et de leur mise en œuvre. La décentralisation d'un système politique suppose l'existence d'arènes de pouvoir autonomes les unes par rapport aux autres, qui sont autant d'espaces potentiels permettant le développement de tels conflits d'interprétation, mais aussi de formes contrastées de mise en œuvre.

Mots clés

Politique de formation, politique de l'emploi, chômage, région, Suisse

Journal of Economic Literature: I 68 Public Policy ; J 64 Unemployment 